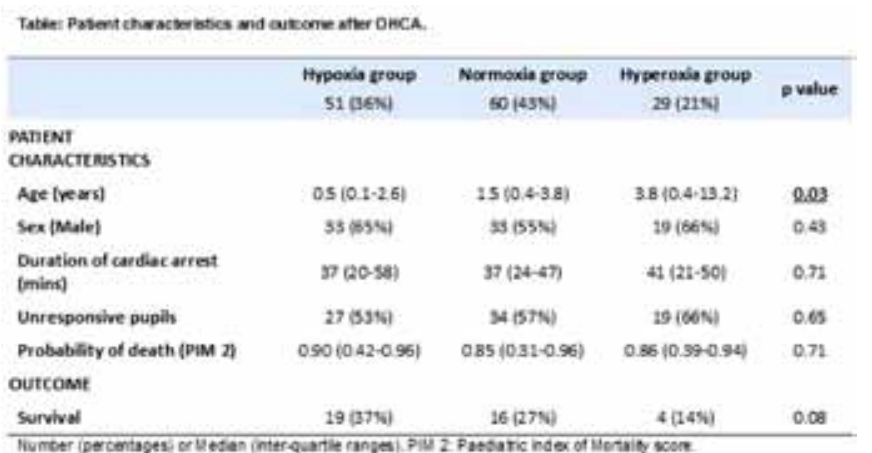

Conclusions This study has observed a difference in survival related to oxygen tension status, with a trend to worsening survival from hypoxia through to hyperoxia. Confirmation of this preliminary finding is required in a larger cohort before embarking on a randomised controlled trial.

\section{HYPOCORTISOLEMIA IN SICK CHILDREN ON PAEDIATRIC INTENSIVE CARE UNIT (PICU): TRANSIENT OR CAUSE FOR CONCERN}

doi:10.1136/archdischild-2012-302724.0807

${ }^{1} \mathrm{H}$ Bentur, 'S Mahoney, ${ }^{2} \mathrm{~J}$ Blair. 'Paediatric Intensive Care; ${ }^{2}$ Paediatric Endocrine, AlderHey Children's NHS Foundation Trust, Liverpool, UK

Background and Aims Cortisol insufficiency has been reported in sick children with severe sepsis, post-cardiac surgery, and may contribute to rapid cardiovascular collapse. Hypothalamic-pituitaryadrenal axis dysfunction may play a role in low-cardiac-output syndrome. We performed a review of PICU patients to describe cortisol levels in those with suspected adrenal insufficiency.

Methods Retrospective review of PICU patients (general and cardiac cases) over 6 months from April to September 2011, who had cortisol levels checked and/or received hydrocortisone.

Results Total PICU admissions were 519, of which $30(5.7 \%)$ patients had cortisol levels. Most common indication for cortisol assessment was refractory hypotension (73\%) in cardiac and sepsis patients. $12 / 30(40 \%$ ) had cortisol levels $<500 \mathrm{nmol} / \mathrm{L}$ (suboptimal response to stress), and $2 / 12$ had undetectable cortisol levels. Of 12 with low cortisol, $50 \%$ were post-operative cardiac neonates and $50 \%$ were mix of other post-operative and chronic illness, $75 \%$ were hypotensive on inotropes and one-third of these received hydrocortisone in PICU. Hydrocortisone dose used was variable. None of the septic children had low cortisol in our study group. $50 \%$ of hypocortisolemia patients were followed up by endocrine team and had normal cortisol levels on follow-up. 10/30(33\%) patients with suspected adrenal insufficiency died (50\% had hypocortisolemia).

Conclusions Our data shows that hypocortisolemia can be transient in sick PICU patients, and may play a role in low-cardiac-output syndrome. There is a need to identify these complex patients with high mortality, and have a uniform management policy jointly with advice and follow-up by the Endocrine team.

\section{UNPLANNED READMISSION TO THE PAEDIATRIC INTENSIVE CARE UNIT (PICU). CAN IT BE PREVENTED?}

doi:10.1136/archdischild-2012-302724.0808

D White, H Coscrato Bachmayr. Paediatric Intensive Care Unit, Cambridge University Hospitals NHS Foundation Trust, Cambridge, UK

Background and Aims It is known that patients readmitted to the PICU during the same hospitalization have significantly adverse outcomes ${ }^{1}$. Prevention of unplanned readmissions to the Paediatric
Intensive Care Unit (PICU) is a key factor when considering the quality of care received by our patients.

Methods All admissions to the PICU during 2011 were examined and all readmissions to the unit were identified. A readmission was defined as those requiring PICU $<48$ hours following discharge. Patients who were readmitted for an elective procedure within the specified time were discounted.

Results During 2011, 511 children had 615admissions to the PICU. 12 children were readmitted for acute care having deteriorated in the ward setting. 2 of the 12 children required 2 readmissions. 5 of the readmissions took place between April and September. The remaining 9 occurred during October to March.

Conclusions It remains a subject of debate as to whether or not any of the redamissions could have been avoided as hindsight is always easy. With nearly double the amount readmissions occuring during the winter months, it could be argued that the children were discharged prematurely due to bed pressures. This a serious concern and one which we will continue to monitor.

1. Odetola et al 2007 Pediat Crit Care Med. Going back for More.

\section{PROSPECTIVE STUDY OF PEDIATRIC CARDIAC ARREST IN EUROPEAN AND LATINOAMERICAN INTENSIVE CARE UNITS}

doi:10.1136/archdischild-2012-302724.0809

1,2 J del Castillo Peral, ${ }^{3} \mathrm{~J}$ López-Herce, European Latinoamerican Study Group of Cardiac Arrest in Children. 'Servicio de Cuidados Intensivos Pediátricos, Hospital General Universitario Gregorio Marañón; ${ }^{2}$ Red Iberoaemericana de Estudio de la Parada Cardiaca en la Infancia; ${ }^{3}$ Hospital General Universitario Gregorio Marañón, Madrid, Spain

Objective To study the characteristics of cardiac arrest (CA) and the results of resuscitation in pediatric intensive care units (PICU).

Patients and methods: Prospective, international, multicentrical study. Children between 1 month and 18 years who suffered CA in the PICU were included. Sustained return of spontaneous circulation (ROSC) and survival at hospital discharge were analyzed.

Results 304 CA episodes in 250 patients were registered, 96 $(31.6 \%)$ in latinoamerican and $208(68.4 \%)$ in European hospitals. Mean age was 47.9 months, mean weight $16.4 \mathrm{~kg}$ and $55.6 \%$ males. The most common causes of CA were cardiac (35.9\%), respiratory $(31.6 \%)$ and sepsis (15.3\%). ROSC was attained in $68.1 \%$ of the cases but only $40.4 \%$ survived. ROSC was higher in European $75.9 \%$ than in Latinamerican 55.4\% PICUs $(\mathrm{p}=0.001)$. Patients with good neurological scales before CA (PCPC $<=2$ ) had significantly higher ROSC rates $(74.5 \%$ vs $33.3 \%$; $=0.006)$. Patients who had suffered a previous CA had lower ROSC percentages ( $51.7 \%$ vs $71.1 \%$; $\mathrm{p}=0.03$ ). Respiratory and cardiac CA have higher survival rates $(40 \%$ and $56.3 \%)$ than sepsis (13.5\%), and neurologic and traumatic causes $(31.7 \%)(p<0.001)$. Initial respiratory arrests achieved higher survival rates than primary CA ( $49 \%$ vs $35.1 \%$; $p=0.029$ ). Patients previously receiving inotropic drugs survived less than those who received no medication ( $31.3 \%$ vs $58 \%$; p $<0.001$ ).

Conclusions Although $68 \%$ of patients who suffered a CA attained ROSC only $40 \%$ of them survived. Ethiology of CA, type of initial arrest and previous treatment with inotropics influence survival in PICU cardiac arrest.

\section{MORBIDITY AND MORTALITY IN CRITICALLY ILL CHILDREN WITH SPONTANEOUS INTRACEREBRAL HEMORRHAGE}

doi:10.1136/archdischild-2012-302724.0810

S Stabouli, A Violaki, E Volakli, S Kalamitsou, M Kotsiou, M Sdougka. PICU, Hippokration Hospital, Thessaloniki, Greece

Background and Aims Spontaneous intracerebral hemorrhage (ICH) accounts for approximately half of stroke in childhood with 